\title{
Impact of polyphenol-rich green tea extracts on the protection of DOPC monolayer against damage caused by ozone induced lipid oxidation*
}

\author{
Elżbieta Rudolphi-Skórska ${ }^{\bowtie}$, Barbara Dyba, Barbara Kreczmer and Maria Filek \\ Department of Biochemistry, Biophysics and Biotechnology Institute of Biology, Pedagogical University of Cracow, Kraków, Poland
}

Effectiveness of green tea (compared to two single polyphenols) in removing ozone derived reactive oxygen species acting on dioleoylphosphatidylcholine monolayers was determined. Lipid oxidation was followed by changes in mechanical properties of the layer spread on the aqueous subphase containing various amounts of ozone in the presence and absence of polyphenolics. It was shown that the tea extract (containing $8.5 \times 10^{-4}$ $\mathrm{mg} / \mathrm{cm}^{3}$ polyphenols) is capable of inactivating $0.4 \mathrm{ppm}$ ozone. The DPPH radical scavenging test set polyphenols in the order of increasing activity, consistent with their protective effect in relation to lipid oxidation, showing the highest efficacy of EGCG.

Keywords: green tea, oxidative stress, lipid oxidation, cell membrane, ozone-pollutant

Received: 03 January, 2018; revised: 27 April, 2018; accepted: 09 May, 2018; available on-line: 30 May, 2018

e-mail: elzbieta.rudolphi-skorska@up.krakow.pl

*This subject was presented at the 5th European Joint Theoretical/ Experimental Meeting on Membranes (EJTEMM); December 6-8, 2017 Kraków, Poland

Abbreviations: DOPC, 1,2-dioleoyl-sn-glycero-3-phosphocholine; $\mathrm{DPPH}, 1$,1-diphenyl-2-picrylhydrazyl radical; EGCG, epigallocatechin gallate; GA, gallic acid; GT, green tea extract; ROS, reactive oxygen species

\section{INTRODUCTION}

Environmental stresses leading to oxidative stress are the main cause of civilization diseases (cancer, diabetes, heart diseases) (Saeid et al., 2018). Ozone, an air pollutant (a component of smog) is one of the important stressors affecting health (Bell et al., 2014). Being the strongest oxidant (standard redox potential $2.07 \mathrm{~V}$ ), it is able to oxidize many substances including unsaturated lipids forming cell membranes. However, there are relatively few papers focused on the oxidation of lipid monolayers by $\mathrm{O}_{3}$ dissolved in an aqueous medium (Brown et al., 2011; Qiao et al., 2013; Rudolphi-Skórska et al., 2014; Rudolphi-Skórska et al., 2016; Rudolphi-Skórska et al., 2017).

Consequences of lipid oxidation for the structure and properties of biological membranes are discussed in numerous papers (e.g. Solis-Calero et al., 2015). Complexity of native membranes entails that many studies on lipid oxidation are carried out in model systems. The Langmuir trough technique in which the lipid layer and subphase compositions, layer compression rate and degree can be controlled, is frequently and successfully applied for studying lipid layer properties (e.g. Morandat et al., 2003). Configuration of the Langmuir model, in which all participants of processes leading to layer modifica- tions are working in situ, i.e. oxidant reacting with lipid layer and possibly present antioxidants, to some extent reflects the biological systems.

A lot of work has been done on oxidation-preventing action of antioxidants (e.g. Apak et al., 2016; Lu et al., 2010). In this group, polyphenols exhibiting high ability of ROS scavenging occupy a significant place. It was proved that drinking green tea, rich in polyphenols and catechins, has a beneficial effect on human health (Higton \& Frei, 2003). Molecular mechanism of polyphenolic antioxidants action was discussed in many papers (e.g.: Leopoldini et al., 2011).

Classification of antioxidative ability can be done on the basis of various tests which "do not necessarily correlate with each other", as indicated in (Pinchuk et al., 2012). The Langmuir model can be successfully used for a quantitative evaluation of the effectiveness of antioxidants' action.

The aim of this study, which is one in a series on the various physico-chemical aspects of lipid oxidation with ozone (Rudolphi-Skórska et al., 2014; Rudolphi-Skórska et al., 2016; Rudolphi-Skórska et al., 2017), was to quantify the protective effect of green tea (GT) extract and two antioxidants present in it, namely epigallo catechin gallate (EGCG) and gallic acid (GA), against ozone-induced oxidation of the DOPC monolayer. The DOPC chosen for experiments represent the cholines which are present in large quantity in both animal and plant cell membranes (Yeagle, 2016). Because oxidation of unsaturated lipids leads to the creation of water-soluble products, reduction in the amount of material capable of forming a layer was adopted as a measure of the lipid oxidation (RudolphiSkórska et al., 2014; Rudolphi-Skórska et al., 2016; Rudolphi-Skórska et al., 2017). A decrease in the ozone concentration in the subphase after polyphenol addition was also measured. EGCG is the best electron donor among catechins and therefore is the best free radical scavenger (Higdon \& Frei, 2003). Gallic acid, as the simplest polyphenol, was chosen for comparison.

For studied polyphenols was performed also DPPH test for their antioxidative ability.

\section{MATERIALS AND METHODS}

Materials. 1,2-dioleoyl-sn-glycero-3-phosphocholine (DOPC) - Avanti Polar Lipids Inc. (USA/Canada); gallic acid (GA) and epigallocatechin gallate (EGCG) - Sigma; green tea (GT) extract in the form of dietary supplement - Olimp Labs (Poland); 1,1-diphenyl-2-picrylhydrazyl (DPPH) - Sigma; solvents (chloroform, ethanol) of chemical purity - POCh (Poland); freshly deionized water produced by HLP 5 Hydrolab (Poland). 

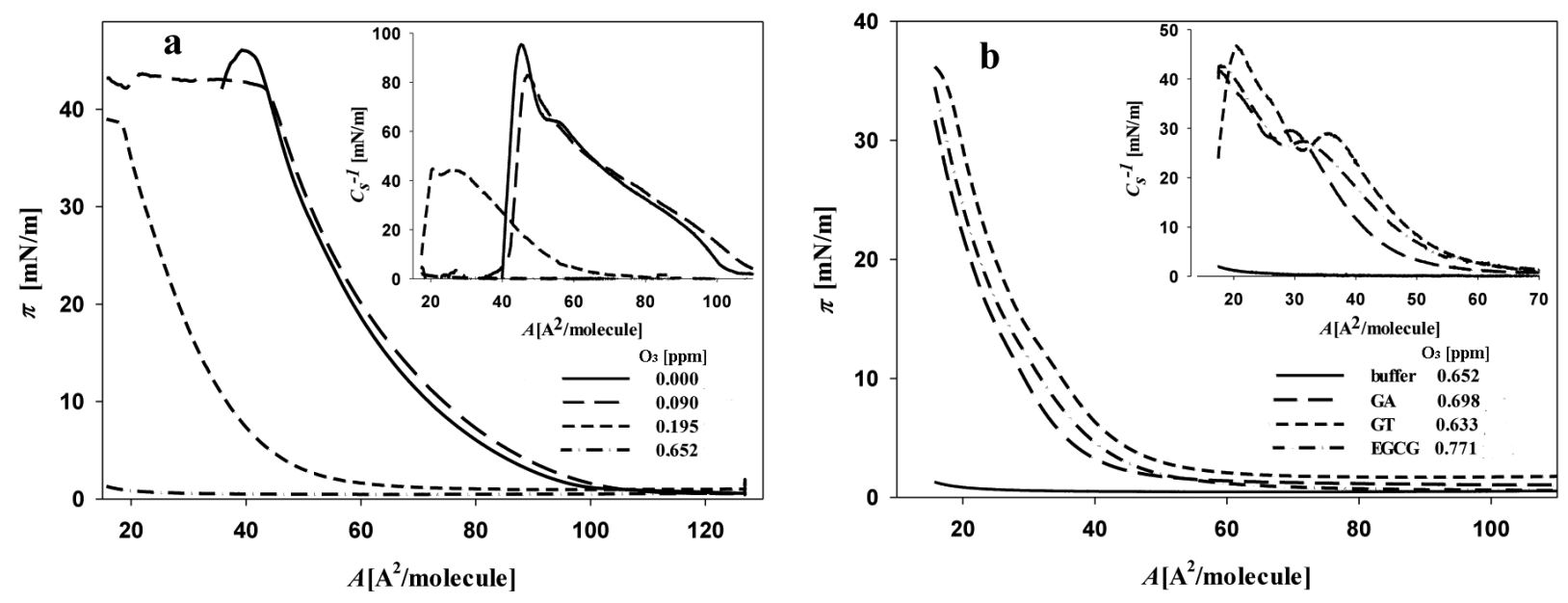

Figure 1. Surface pressure isotherms and corresponding compressibility modulus (inset) of the DOPC layers spread on the subphasecontained the indicated concentrations of ozone dissolved in a pure buffer.

$\mathrm{a}$ - in the absence of antioxidants; $\mathrm{b}$ - in the presence of studied polyphenols $\left(8.5 \times 10^{-4} \mathrm{mg} / \mathrm{cm}^{3}\right)$ added to the ozone containing buffer.

Buffer Ozonation. Ozone was produced from pure oxygen by ozone generator FM 500 (Grekos, Poland). Phosphate buffer $(0.01 \mathrm{M}, \mathrm{pH} 7)$ was saturated with ozone and its specified concentration was obtained by appropriate dilution. Ozone concentration in a buffer was determined from discoloration of the indigo trisulfonate carmine, according to (Bader \& Hoigne, 1981). Taking into account fast ozone decomposition (Gurol \& Singer, 1982), samples for analysis were taken at the moment of lipid deposition.

Surface Pressure Isotherms. Langmuir trough (KSV, Finland) of the surface area of $243 \mathrm{~cm}^{2}$ was used for surface pressure isotherm registration at constant compression rate corresponding to $5 \mathrm{~mm} / \mathrm{min}$ barrier speed. Lipid monolayer was formed by spreading a defined amount of DOPC chloroform solution at $1 \mathrm{mg} / \mathrm{cm}^{3}$ concentration on the subphase composed of aqueous $0.01 \mathrm{M}$ phosphate buffer, $\mathrm{pH}$ 7.0. Surface tensions were measured witha Pt-Wilhelmyplate. Experiments were performed at $25^{\circ} \mathrm{C} \pm 1^{\circ} \mathrm{C}$.

Anti-DPPH radical activity determination. Antioxidative activities of polyphenolic samples (of mass concentrations in the range between 0 and $0.02 \mathrm{mg} /$ $\mathrm{cm}^{3}$ ) were tested in the reaction with $\mathrm{DPPH}$ (at constant concentration of $9.2 \times 10^{-5} \mathrm{M}$ ) in methanol. Polyphenol equivalent concentration in the Green tea extract was calculated based on the data from the certificate of the productused. The decrease in absorbance of DPPH solution was continuously registered for 3-5 $\mathrm{min}$ at room temperature. Antioxidant activity - Q (quenching) was represented by the relative reduction in DPPH concentration $Q(t)=\left[A_{0}-A_{a}(t)\right] / A_{0}$, where $A_{0}$ is the initial absorbance of the DPPH solution (at $517 \mathrm{~nm}$ ), $\mathrm{A}_{\mathrm{a}}(\mathrm{t})$ is time dependent absorbance of the reaction mixture and $t-$ time. Changes in time of the $\mathrm{Q}$ parameter during the first $60 \mathrm{~s}$ were fitted to an exponential function whose slope extrapolated to time 0 was taken as a measure of the initial reaction rate.

\section{RESULTS AND DISCUSSION}

The protective effect was determined by comparing the responses of DOPC layers to ozone dissolved in the subphase in the presence and absence of the studied polyphenols, and by changes in the ozone concentration in the aqueous phase after their addition. The influence of ozone on physicochemical properties of DOPC layers was detected by measuring surface pressure isotherms as shown for a layer spread on antioxidant-free buffer solution.

The appearance of oxidation products, including low-molecular species, strongly influences lipid layer structure and properties (Brown et al., 2011; Reis \& Spickett, 2012; Jurkiewicz et al., 2012). Soluble oxidation products, when transferred to the aqueous phase, reduce the amount of material capable of forming amono layer causing significant disruption of the lipid layer structure. This results in a modification of the dependence of the layer surface pressure and consequently in compression modulus on area per molecule. With an increase in the ozone concentration, the surface pressure isotherms shift towards the lower values of nominal area per molecule due to a decreased amount of material capable of insoluble layer forming (Fig. 1a). The maximal values of compression modulus decrease (inset in Fig 1a): at the 0.195 ppm $\mathrm{O}_{3}$ this value drops more than $50 \%$ relative to that obtained for the layer not exposed to ozone (95.3 $\mathrm{mN} / \mathrm{m}$ to $43.1 \mathrm{mN} / \mathrm{m}$ ). Compression module represents layer resistance $v s$. compression, informing about its rigidity. Lipid oxidation significantly reducing this quantity may have consequences for layer permeability. Such strong changes in the mechanical properties of the lipid layer, related also to modified interlayer interactions, may be of key importance for cellular processes (Makky \& Tanaka, 2015).

Under applied experimental conditions, the presence of polyphenolic antioxidants does not influence the surface pressure isotherm of the DOPC layer deposited on a buffer in the absence of ozone, but noticeably increases the threshold initial ozone concentrations leading to visible changes in surface pressure isotherms. In Fig. 1b, surface pressure isotherms of the DOPC layers exposed to comparable initial ozone concentrations in subphases in the absence and presence of antioxidants are shown. In these experiments, the polyphenol content was set at a constant mass concentration $\left(8.5 \times 10^{-4} \mathrm{mg} / \mathrm{cm}^{3}\right)$ corresponding to the polyphenol amount equivalent to $5 \times 10^{-6} \mathrm{M}$ GA. This mass concentration of EGCG is in a $1.9 \times 10^{-6} \mathrm{M}$ solution. It is worth noting that the ratio of the used molar concentrations of GA to EGCG is inversely proportional to the ratio of the number of $\mathrm{OH}$ 

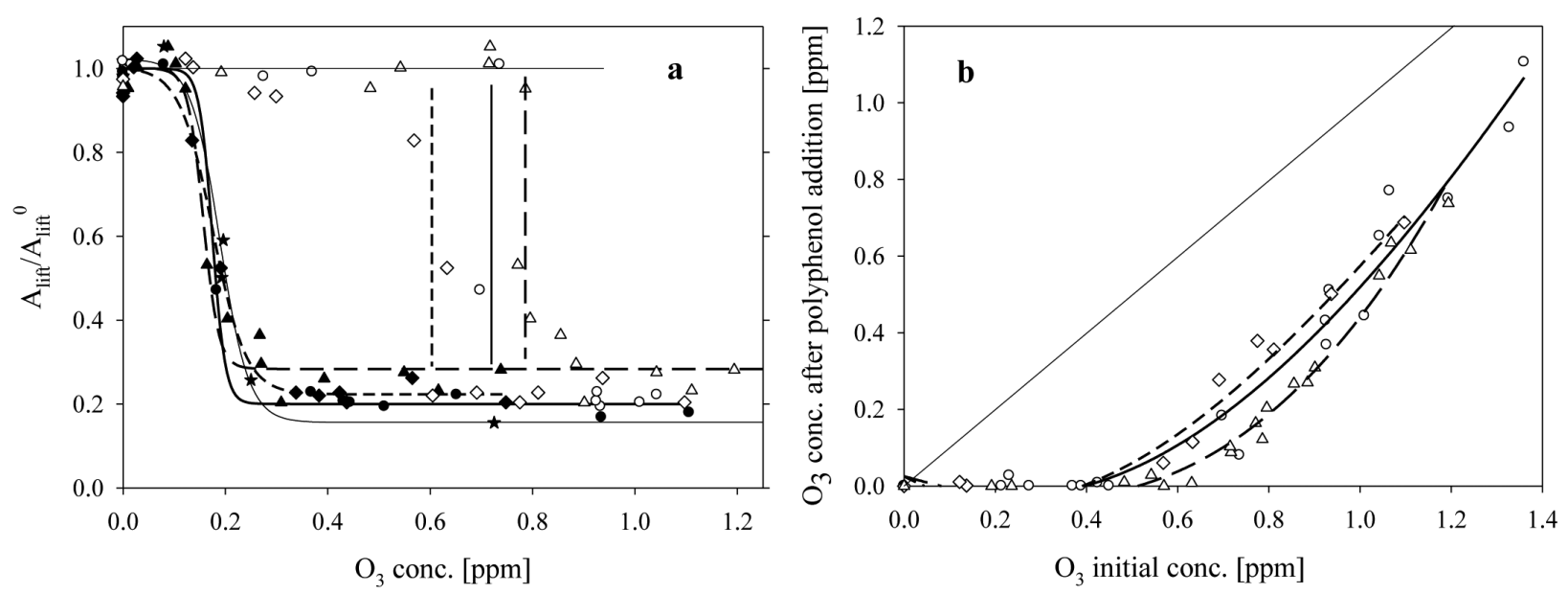

Figure 2a. $A_{\text {lift }} / A_{\text {lift }}{ }^{0}$ parameter representing a layer-forming capacity of DOPC spread on the subphase in the absence (full stars and thin solid line) and in the presence of the studied polyphenols, as a function of the ozone concentrations.

Circles and thick solid lines - GA, up triangles and long dash lines - EGCG, diamonds and short dash lines - GT extract. The open symbols of the same shapes represent the dependence of this parameter on the initial ozone concentrations, before the antioxidants' addition. Vertical lines indicate the shift of threshold levels of ozone, caused by its reaction with antioxidants.

Figure $2 \mathrm{~b}$. Dependence of ozone concentration after addition of polyphenols (at mass concentration equal to $8.5 \times 10^{-4} \mathrm{mg} / \mathrm{cm}^{3}$ ) on initial ozone level in a buffer.

Thin straight line of slope 1 illustrates the situation in which the concentration of ozone would not change under the influence of added antioxidants. The lines denote nonlinear fits of experimental data.

groups responsible for the reaction with an oxidant (3:8). In the absence of polyphenols, isotherm of the DOPC layer on a buffer containing about $0.7 \mathrm{ppm} \mathrm{O}_{3}$ indicates that the number of molecules capable of layer formation was considerably reduced. When lipid contacted a buffer of similar initial ozone concentration but with added antioxidants, the destruction of the layer was not as big and the surface pressure isotherm was registered in the same range of nominal areas per molecule.

Changes in the layer-forming capacity caused by lipid oxidation were expressed by the ratio of the areas per molecule at which the surface pressure increased by $1 \mathrm{mN} / \mathrm{m}$ (named lift-off area $A_{\text {lift }}$ ) for layers exposed to ozone, relative to the value obtained for non-oxidized lipid $\left(\mathrm{A}_{\text {lift }}{ }^{0}\right)$. This quantity can be taken as a measure of lipid oxidation (Rudolphi-Skórska et al., 2014).

Rapid ozone decomposition precludes its accurate dosing, thus to get reliable data the experiments were done in the entire range of its level. The results obtained for all tested substances are shown in Fig. 2a. As wehave shown previously (Rudolphi-Skórska et al., 2014; Rudolphi-Skórska et al., 2016; Rudolphi-Skórska et al., 2017), the dependence of the ratio of the lift-off area values on the ozone concentration has a characteristic S-shape. The range of ozone concentrations corresponding to an abrupt decrease in this value can be identified as threshold oxidant concentration above which significant destruction of lipid layer occurs. Further increase in the oxidant content does not influence the values of the lift-off areas ratio and this constant plateau value can be interpreted as a measure of the maximum loss of lipid material due to oxidation.

The dependencies presented in Fig. 2a show that plateau $\left(A_{\text {lift }} / A_{\text {lift }}{ }^{0}\right)$ value for DOPC layers spread on thesubphase that do not contain any antioxidants equals to 0.16. The presence of polyphenols only slightly affects this value. The strongest effect is observed for EGCG (0.28) which may mean that its relatively large molecular size and possibly its oxidation products can stabilize the lipid layer to some extent.
In contrast to changes in the plateau values of $\left(\mathrm{A}_{\mathrm{ijft}} /\right.$ $\left.A_{\text {lift }}{ }^{0}\right)$ ratio, the antioxidant's presence in the subphase significantly affects the threshold initial ozone concentration (before polyphenol addition) at which the state of lipid layer rapidly changes. Shifting the sudden change of $\left(\mathrm{A}_{\text {lift }} / \mathrm{A}_{\text {lift }}{ }^{0}\right)$ ratio towards higher initial ozone concentrations suggests that the role of polyphenols present in the subphase relies on reducing the amount of ozone-derived ROS. This was confirmed by performing measurements of the ozone concentrations in a given buffer before and after addition of the test substances. In Fig. 2b, concentrations of ozone in solutions containing constant amount of the studied polyphenols $\left(8.5 \times 10^{-4} \mathrm{mg} / \mathrm{cm}^{3}\right)$ as a function of the initial (before antioxidant addition) ozone level are presented. The deviations of experimental points from the line of unit slope give an idea of the amount of ozone consumed in the bulk reaction. EGCG seems to be the most effective in ozone depletion from aqueous solutions, whereas actions of the GT extract and GA were similar.

Presentation of $A_{\text {liff }} / A_{\text {lift }}{ }^{0}$ ratio as a function of ozone concentration after antioxidant addition, i.e. the concentration that effectively acts on the lipid layer (Fig. 2a), shows that the threshold ozone levels for all tested systems are very similar, which means that polyphenols do not influence the lipid oxidation reaction and their role is to reduce the amount of ROS.

Action of water-soluble polyphenols significantly differs from that of antioxidants present in the hydrophobic part of the membranes. As shown in (Rudolphi-Skórska et al., 2014; Rudolphi-Skórska 2016), $\alpha$-tocopherol added to galactolipids has little effect on the threshold ozone concentration, causing a destruction of the mixed layer. On the other hand, tocopherol and its ionized oxidation products are able to stabilize the structure of oxidized layer which is manifested by an increase in the $\mathrm{A}_{\text {lift }} / \mathrm{A}_{\text {lift }}{ }^{0}$ plateau level.

The lipid layers are a sensitive detector of the amount of ozone in the aqueous subphase (Rudolphi-Skórska et al., 2014; Rudolphi-Skórska et al., 2016; Rudolphi-Skórska et al., 2017). The obtained results (Fig. 1 and 2a) show 

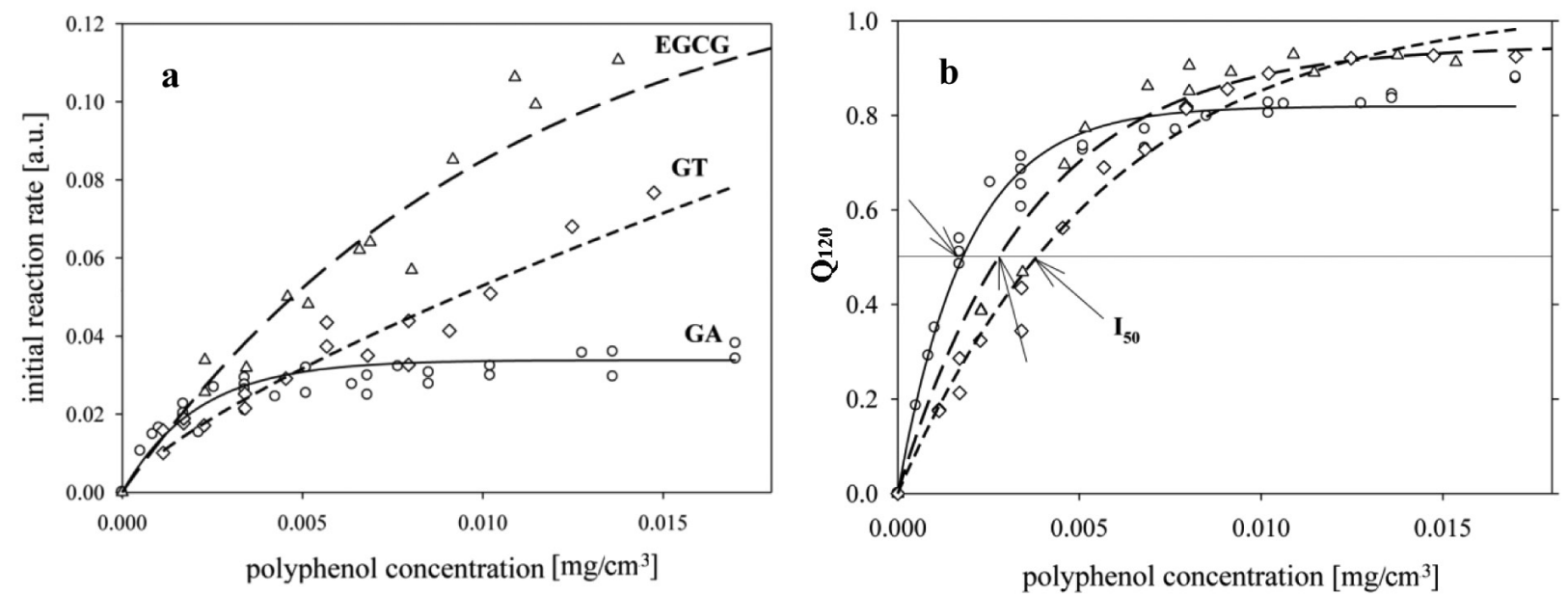

Figure 3a. The initial DPPH/polyphenols reaction rate as a function of polyphenol concentration.

Figure $3 \mathrm{~b}$. Fraction of radical concentration after $120 \mathrm{~s}$ of reaction duration as a function of polyphenol concentration.

The arrows indicate concentrations of polyphenols that reduce the radicals' level by half. (Data collected in Table). The lines denote nonlinear fit of experimental data with $R_{\text {sqr }}$ values equal to $0.88,0.94$ and 0.95 for GA, GT and EGCG, respectively.

Table 1. Concentrations of polyphenols reducing the radicals' level by half after $120 \mathrm{~s}$ of reaction duration $\left(\mathrm{I}_{50}\right)$.

\begin{tabular}{lccc}
\hline Tested substance & GA & EGCG & GT extract \\
\hline $\mathrm{I}_{50}\left[\mathrm{mg} / \mathrm{cm}^{3}\right]$ & $1.8 \times 10^{-3}$ & $2.8 \times 10^{-3}$ & $3.85 \times 10^{-3}$
\end{tabular}

that with increasing ozone concentration, the lipid layer undergoes degradation associated with a reduction of layer rigidity. At higher oxidant levels, the lipid oxidation finally results in layer destruction caused by the loss of material forming a monolayer. Membranes exposed to such strong oxidizing conditions may even lose their continuity (Cwiklik \& Jungwirth, 2010). The obtained dependencies of the $\mathrm{A}_{\text {lift }} / \mathrm{A}_{\text {lift }}{ }^{0}$ ratio on the ozone concentration allow to quantify and compare the action of the tested antioxidants which, by reacting with ozone generated oxidizing radicals, reduce their concentration. Qualitatively, all tested antioxidants exhibit a similar protective effect. Quantitatively, considering the amount of ozone removed from the subphase and the resulting shift of threshold ozone level for lipid layer degradation, the studied polyphenolic antioxidants can be ranked according to their increasing effectiveness: GT extract, GA and EGCG being the most effective. The least effect of GT is probably related to the fact that this natural product, besides the phenolic compounds also contains other substances whose presence may influence the reaction with ROS.

The results presented above on the effectiveness of GA, EGCG and GT in scavenging ozone derived ROS were compared with their reaction with the DPPH radical. The initial rates of this reaction, as shown in the Fig. 3a, change in different ways with the concentration of the studied polyphenols (at constant DPPH level).

Another way of assessment of antiradical ability is based on the concentration dependence of the $Q_{t}$ parameter (describing a fraction of radicals remaining after a defined reaction time) (Fig. 3b). Antioxidant concentrations decreasing the DPPH radical amount to half $\left(\mathrm{I}_{50}\right)$ after a 2 min reaction, obtained from the dependencies presented in Fig. 3b, are collected in Table 1. For a given antioxidant, the $I_{50}$ parameter, representing a different stage than the initial rate of the reaction, is independent of the concentration but depends on the reaction time. As such, it cannot be considered as a universal param- eter describing an antioxidant activity. It will depend on the reaction kinetics, which can be very different for different substances (Xie \& Schaich, 2014).

As one can see (Fig. 3 and Table 1), information obtained from both factors shows that polyphenolic antioxidant concentrations necessary for effective neutralization of the DPPH radicals have to be much higher $\left(8.5 \times 10^{-4}\right.$ $\mathrm{mg} / \mathrm{cm}^{3}$ ) in comparison to ozone-derived ROS in aqueous solutions (as it was shown in experiments with lipid model). This means that information on the antioxidant effectiveness based on the results of the DPPH test cannot be simply transferred to their action $v s$. other radicals (e.g. ROS) in a different environment (alcoholic vs. aqueous).

\section{CONCLUSIONS}

A quantitative assessment of the effects of oxidation (layer destruction) and the antioxidative activity of some polyphenolics was made on the basis of results obtained in a model system in which a single, quantified stress factor $\left(\mathrm{O}_{3}\right)$ acts on the biomolecules (lipid layers) in the presence of tested antioxidants. The system arrangement, at least qualitatively, reflects a situation in cells where all participants of these processes operate in the same place. The efficiency of operation of polyphenolic substances determined this way is free of screening or amplifying effects which may come from metabolic processes taking place in the living system (when tests are conducted on whole cells or whole organisms).

The protective ability, defined by the shift of the ozone threshold level (separating non-destructive from the destructive range of concentrations of the oxidant acting on the lipid layer) allows the studied polyphenols to be ranked according to their effectiveness: EGCG> GA $>$ GT. EGCG also exhibited the greatest activity in DPPH radical inactivation.

It was shown that at a low concentration $\left(8.5 \times 10^{-4}\right.$ $\mathrm{mg} / \mathrm{cm}^{3}$ of polyphenolic species), the GT extract can 
deactivate 0.4 ppm ozone in a solution. Thus, drinking green tea may constitute an effective therapeutic and preventive measure in the widely understood protection of the health of societies.

\section{Conflicts of interest}

The authors declare no conflicts of interest.

\section{REFERENCES}

Apak R, Ozyurek M, Guclu K, Capanoglu E (2016) Antioxidant activity/capacity measurment. 1. Classification, physicochemical principles, mechanisms and electron transfer (ET)-based assays. I Agric Food Chem 64: 997-1027. http://doi.org/10.1021/acs.jafc.5b04739

Bader H, Hoigne J (1981) Determination of ozone in water by the indigo method. Water Res 15: 449-456. http://doi.org/10.1016/00431354(81)90054-3

Bell ML, Zanobetti A, Dominici F (2014) Systematic reviews and meta- and pooled analyses who is more affected by ozone pollution? A systematic review and meta-analysis. Am J Epidemiol 180: 15-28. https://doi.org/10.1093/aje/kwu115

Brown SHJ, Mitchell TW, Blanksby SJ (2011) Analysis of unsaturated lipids by ozone-induced dissociation. Biochim Biophys Acta 1811: 807817. http://doi.org/10.1016/j.bbalip.2011.04.015

Cwiklik L, Jungwirth P (2010) Massive oxidation of phospholipid membranes leads to pore creation and bilayer disintegration. Chem Phys Lett 486: 99-103. https://doi.org/10.1016/j.cplett.2010.01.010

Gurol MD, Singer PC (1982) Kinetics of ozone decomposition: a dynamic approach. Environ Sci Technol 16: 377-383. https://doi. org/10.1021/es00101a003

Higdon JV, Frei B (2003) Tea catechins and polyphenols: Health effects, metabolism, and antioxidant functions. Crit Rev Food Sci Nutr 43: 89-143. https://doi.org/10.1080/10408690390826464

Jurkiewicz P, Olzynska A, Cwiklik L, Conte E, Jungwirth P, Megli FM, Hof M (2012) Biophysics of lipid bilayers containing oxidatively modified phospholipids: insights from fluorescence and EPR experiments and from MD simulations. Biochim Biophys Acta 1818: 2388-2402. https://doi.org/10.1016/j.bbamem.2012.05.020

Leopoldini M, Russo N, Toscano M (2011) The molecular basis of working mechanism of natural polyphenolic antioxidants. Food Chem 125: 288-306. https://doi.org/10.1016/j.foodchem.2010.08.012

Lu J-M, Lin PH, Yao Q, Chen Ch (2010) Chemical and molecular mechanisms of antioxidants: experimental approaches and mod- el systems. I Cell Mol Med 14, 840-860. https://doi.org/10.1111 j.1582-4934.2009.00897

Makky A, Tanaka M (2015) Impact of lipid oxidization on biophysical properties of model cell membranes. J Phys Chem B 119: 5857-5863. http://dx.doi.org/10.1021/jp512339m

Morandat S, Bortolato M, Anker G, Doutheau A, Lagarde M, Chauvet J-P, Roux B (2003) Plasmalogens protect unsaturated lipids against UV-induced oxidation in monolayer. Biochim Biophys Acta 1616: 137 146. http://dx.doi.org/10.1016/j.bbamem.2003.08.001

Pinchuk I, Shoval H, Dotan Y, Lichtenberg D (2012) Evaluation of antioxidants: scope, limitations and relevance of assays. Chem Phys Lipids 165: 638-647. https://doi.org/10.1016/j.chemphyslip.2012.05.003

Qiao L, Ge A, Osawa M, Ye S (2013) Structure and stability studies of mixed monolayers of saturated and unsaturated phospholipids under low-level ozone. Phys Chem Chem Phys 15: 17775-17785. https:// doi.org/10.1039/C3CP52484A

Reis A, Spickett CM (2012) Chemistry of phospholipid oxidation. Biochim Biophys Acta 1818: 2374-2387. https://doi.org/10.1016/j.bbamem.2012.02.002

Rudolphi-Skórska E, Filek M, Zembala M (2014) Physicochemical aspects of reaction of ozone with galactolipid and galactolipid-tocopherol layers. J Membr Biol 247: 639-649. https://doi.org/10.1007/ s00232-014-9681-9

Rudolphi-Skórska E, Filek M, Zembala M (2016) Tocopherol/gallic acid cooperation in the protection of galactolipids against ozone-induced oxidation. J Membr Biol 249: 87-95. https://doi.org/10.1007/ s00232-015-9851-4

Rudolphi-Skórska E, Filek M, Zembala M (2017) The effects of the structure and composition of the hydrophobic parts of phosphatidylcholine-containing systems on phosphatidylcholine oxidation by ozone. J Membrane Biol 250: 493-505. https://doi.org/10.1007/ s00232-017-9976-8

Saeid G, Huige L, Ismail L (2018) Oxidative stress: a unifying mechanism for cell damage induced by noise, (water-pipe) smoking, and emotional stress - therapeutic strategies targeting redox imbalance. Antiox Redox Sign. https://doi.org/10.1089/ars.2017.7257

Solís-Calero C, Ortega-Castro J, Frau J, Muñoz F (2015) Nonenzymatic reactions above phospholipid surfaces of biological membranes: reactivity of phospholipids and their oxidation derivatives. Oxid Med Cell Longev 2015: 22. https://doi.org/10.1155/2015/319505

Xie J, Schaich KM (2014) Re-evaluation of the 2,2-diphenyl-1-picrylhydrazyl free radical (DPPH) assay for antioxidant activity. I Agric Food Chem 62: 4251-4260. https://doi.org/10.1021/jf500180u

Yeagle PL (2016) The Membranes of Cells. Academic Press. 012800486X, 9780128004869 